\title{
Results of Auditory Training for Blind and Visually Handicapped Children and Adolescents
}

\author{
E. Bogusz-Witczak*, E. Skrodzka, A. Furmann, E. Hojan and K. Przybek \\ Institute of Acoustics, A. Mickiewicz University, Umultowska 85, 61-614 Poznań, Poland
}

\begin{abstract}
Results of an auditory training for blind/visually impaired children and teenagers are presented. A measure of the training effectiveness is the difference between the results of a pre- and post-training verification test. Two age groups of visually handicapped young persons were tested: 7-12 years old and 14-19 years old. It was shown that the training may be beneficiary for blind or visually impaired children and teenagers, especially in tasks related to localization of moving sound sources. No differences in training results were found between age groups. The results of trained groups were compared to those obtained for not trained control groups of young blind individuals.
\end{abstract}

DOI: $10.12693 /$ APhysPolA.127.117

PACS: $43.66 . \mathrm{Yw}, 43.66 . \mathrm{Hg}, 43.66 . \mathrm{Jw}, 43.66 . \mathrm{Qp}$

\section{Introduction}

As the blind and visually handicapped people cannot make use or full use of the sense of vision, they must rely on the second most important human sense, the sense of hearing. In particular they should be taught how to safely and independently navigate in an urban environment, which is very important in preventing them from social exclusion. Therefore they should be taught to focus auditory attention on small differences in parameters of acoustic waves, which is essential for independent and correct interpretation of environment by hearing and listening.

The aim of the paper is the assessment of effectiveness of auditory training (AT) addressed to blind or visually impaired children and teenagers. The concept and details of auditory training have been comprehensively described in our previous papers [1-4]. Therefore, we recall the most important facts only. The training comprises psychoacoustic and lateralization tasks, like pitch discrimination of tones, noise bands, FM signals, pitch memory; loudness discrimination of tones, noise bands and AM signals; lateralization of tonal and noise signals; timbre discrimination of harmonic signals; simultaneous categorization of pitch and timbre and signal-in-noise detection. The lateralization tasks involve binaural audio reproduction via headphones, while the remaining experiments are based on monaural audio reproduction using a single headphone in the preferred ear. Sounds were presented on a comfortable level of $65 \mathrm{~dB}$ SPL. The method of constant stimuli was used. For each subject twenty training sessions were executed, each lasting $40 \mathrm{~min}$.

The AT is addressed mainly to children and teenagers because they are a promising and potentially the most beneficiary group of the blinds, as people who are congenitally blind or who have lost their sight at an early age are better at identifying and differentiating sounds than

*corresponding author; e-mail: ebogusz@amu.edu.pl those who have become blind in adolescence or adulthood [5].

\section{Pre- and post-test (verification test)}

To be able to evaluate the effect of AT, the auditory training verification test was prepared. It is described in details in our previous paper [2], so it will be just briefly recalled. The verification test is composed of 6 tasks checking the pitch discrimination (task 1), pitch and timbre categorization (task 2), pitch memory (task 3 ), lateralization of a stationary sound source (task 4), lateralization of a moving sound source (task 5), and lateralization of two moving sound sources (task 6). The tasks come from AT and experiments described in $[1,3,4]$. The sound material was presented at a level of $65 \mathrm{~dB}$ SPL via open headphones Sennheiser HD600. The test apparatus and commands were the same as those used in AT [2]. The verification test takes $45 \mathrm{~min}$.

\section{Subjects}

The experiment was performed on 35 participants, 12 blind and 23 visually handicapped, from the school for the blind and visually handicapped in Owińska and Łódź. The group subjected to the acoustic training comprised 6 children of 7-12 years of age ( 5 girls and 1 boy; 3 of whom were blind and 3 visually impaired; mean age 10 years) and 10 teenagers of $14-19$ years of age (5 girls and 5 boys, 5 of whom were blind; mean age 16 years). The control group comprised 9 children aged 7-12 (2 girls and 7 boys, all visually handicapped; mean age 11 years) and 10 teenagers aged 14-19 (3 girls and 7 boys, 4 of whom were blind; mean age 16 years). All participants were otologically normal persons; their hearing loss did not exceed $25 \mathrm{~dB} \mathrm{HL}$ for the frequencies from the range 250-8000 Hz.

\section{Results and analysis of the pre- and post-training verification test}

Each participant was asked to perform the test twice. The participants from the group subjected to the acoustic training performed the test immediately before the 
training and after the four-week lasting training, while the persons from the control group were asked to perform the test twice, at the four-week interval. The results were subjected to multiple factor analysis of variance. Analysis of all results for the two groups was performed taking into account three factors: the type of test (pre-training test, post-training test or the first and the second test), action taken (participation in the acoustic training or not) and type of task performed in the test. The variance analysis (Snedecor $F$-Test, $p$-significance level, $F$-variance ratio [6]) proved a statistically significant difference between the results of pre-training test and post-training test $(F(1,408)=4.138 ; p=0.0425)$ as well as between the results of particular tasks in the test $(F(5,408)=8.453 ; p<0.001)$. Statistically significant was also the interaction between the action (AT) and the type of test $(F(1,408)=6.312, p=0.012)$, which means that the results of a given type of test significantly depended on the participation in the training or not.
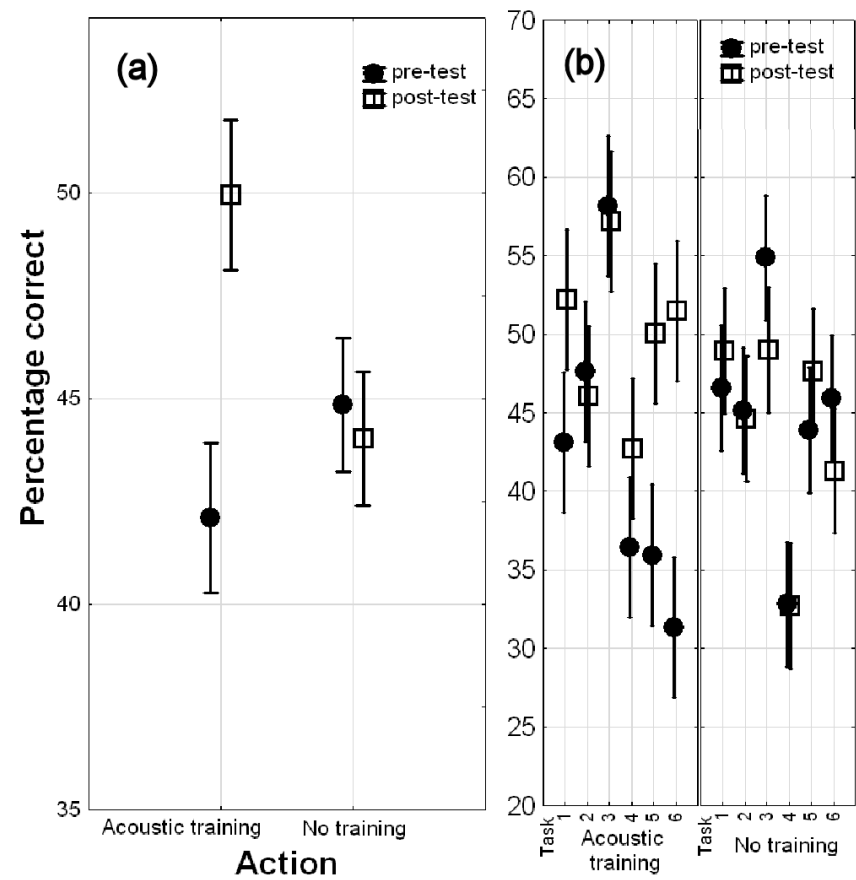

Fig. 1. Averaged results of pre- and post-training verification test for (a) acoustically trained and control groups and (b) acoustically trained and control groups for particular verification tasks.

Figure 1a presents the results of the pre-training and post-training test or the first and the second test versus the action taken so for the persons subjected to the training and for the control group. Vertical bars in all figures indicate standard error. As follows from analysis of results obtained in the post-training test or the second test for the persons participating in the training and for the control group, the persons subjected to AT performed much better than those not subjected to it, $(F(1,204)=5.102 ; p=0.025)$, and the difference is by

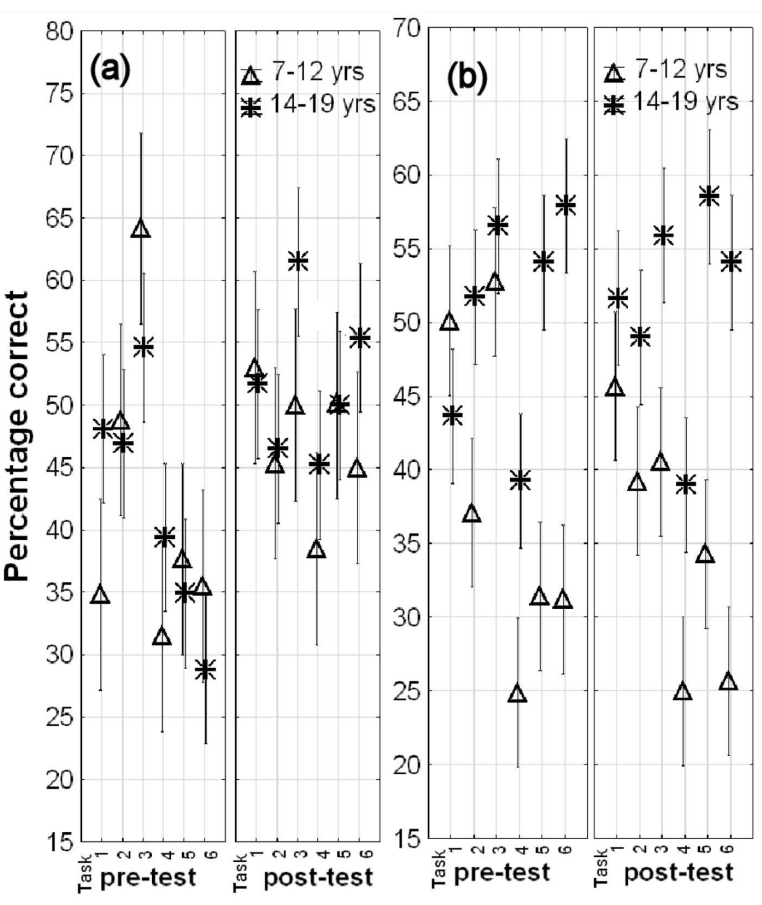

Fig. 2. Averaged results of pre- and post-training verification test for (a) the acoustically trained subjects from both age groups and (b) no training subjects, for both age groups.

6 percentage points. For the control group the results of the first and second test are the same, Fig. 1a.

Analysis of the results of individual tasks and the two groups of participants (Fig. 1b) reveals that in the group subjected to AT, the training caused the greatest difference in realisation of tasks 5 and 6 . The difference in realisation of task 5 was 13 percentage points (the difference significant at the level $p=0.025$, according to the NIR Fisher test [6]), while in realisation of task 6 the difference reached 20 percentage points (the difference significant at the level $p=0.001$, according to the NIR Fisher test). The difference was also noted in the realisation of task 1 , but it was not statistically significant, see Fig. 1b.

As follows from Fig. 2a, for the group subjected to acoustic training no difference in the results was noted between the age groups.

Both in the first and second test, the results of the teenagers from the control group were better than those of the younger children from the control group, Fig. $2 \mathrm{~b}$. Analysis by the post-hoc NIR Fisher test [6], (Statistica $10)$, proved that these differences were statistically significant for realisation of the second task $(p=0.012)$, fourth task $(p=0.003)$, fifth task $(p<0.001)$ and sixth task $(p<0.001)$, Fig. 2b. These differences can be explained by the higher auditory competences (auditory attention, auditory scene analysis) of the older group of teenagers than the young children. 


\section{Conclusions}

Analysis of the above presented results has allowed to draw the following conclusions regarding the effectiveness of AT used.

1. The results of post-training verification test were much better for the acoustically trained participants than for the control group, no differences between the acoustically trained and untrained groups were noted in the pre-training test. For participants of the control groups, no differences between the pre- and post-verification tests were noted.

2. The test results were the same for the younger and older groups of participants subjected to the acoustic training, so the training was beneficial for both age groups.

3. The acoustic training improved the abilities of lateralisation of the moving sound sources, which is directly connected to improved safety of blind or visually impaired people in urban environment.

\section{Acknowledgments}

The work was supported by the National Science Centre (Grant no. 2012/05/B/HS6/03863).

\section{References}

[1] E. Bogusz, H. Koprowska, E. Skrodzka, Acta Phys. Pol. A 121, A19 (2012).

[2] E. Skrodzka, M. Maciągowski, A. Furmann, Acta Phys. Pol. A 125, A31 (2014).

[3] E. Bogusz, H. Koprowska, E. Skrodzka, Arch. Acoust. 37, 549 (2012).

[4] A. Furmann, E. Skrodzka, P. Giżewski, Ł. Nowotny, Acta Phys. Pol. A 123, 988 (2013).

[5] C.Y. Wan, A.G. Wood, D.C. Reutens, S.J. Wilson, Neuropsychologia 48, 344 (2010).

[6] M. Rabiej, Statistics with Statistica Program, Helion, Gliwice 2012 (in Polish). 